\title{
The effectiveness of worry exposure in treating generalized anxiety disorder
}

\author{
Ghasem Shahmoradi $^{\mathrm{a}^{*} \text {, Mohsen Dadashi }}{ }^{\mathrm{b}}$, Zakaria Eskandari ${ }^{\mathrm{c}}$, Mohammad Reza Iravani ${ }^{\mathrm{d}}$, Samaneh \\ Salimi $^{\mathrm{e}}$ and Hajar Jannesari ${ }^{\mathrm{f}}$
}

${ }^{a}$ MSc of Clinical Psychology, Psychology Group, School of Education and Psychology, Tabriz University, Tabriz, Iran.

${ }^{b}$ Phd student of clinical psychology, Tehran University of Medical Sciences, Tehran, Iran

${ }^{c}$ MSc in clinical psychology, Shahid Beheshti University of Medical Sciences, Tehran, Iran

${ }^{d}$ Assistant Professor, Department of Social Work, Islamic Azad University Khomeinishahr Branch, Daneshjou Blvd, Iran

${ }^{e}$ MS Student, Counseling Department, Psychological and Education Science Faculty University of Isfahan, Isfahan, Iran

${ }^{f} M S$ Student, Counseling Department, Islamic Azad University of Khomeinishahr, Khomeinishahr Branch, Daneshjou Blvd, Iran

\section{H R O N I C L E A B S T R A C T}

Article history:

Received January 12, 2013

Received in revised format

30 April 2013

Accepted 12 May 2013

Available online

May 142013

Keywords:

Worry exposure

Generalized Anxiety Disorder

Cognitive Avoidance

Worry

\begin{abstract}
Worry exposure (WE) is a core of cognitive-behavioral treatment for generalized anxiety disorder (GAD). The present study was carried out to examine the efficacy of WE in treating patients with GAD. Three patients with GAD were selected using Structured Clinical Interview for DSM-IV (SCID) based on disorders axis I. Subjects were selected using purposeful sampling, and underwent the treatment after gaining treatment needs. Multiple baseline experimental single case study was used as the method of the present study. The treatment program was carried out for 8 weekly sessions, with a follow up period of 3 months later treatment ending. Subjects completed Pennsylvania State Worry Questionnaire (PSWQ) and Cognitive Avoidance Questionnaire (CAQ). Research findings represented that WE decreased the intensity of GAD symptoms: Pathologic worry and cognitive avoidance. WE has suitable efficacy in treating Generalized Anxiety Disorder and it maintains suitable efficacy in treating GAD.
\end{abstract}

\section{Introduction}

Generalized anxiety disorder (GAD) is normally defined in the Diagnostic and Statistical Manual of Mental Disorders, Fourth Edition (DSM-IV) as "the presence of persistent, excessive anxiety and worry about a number of events and occurring on more days than not for $>6$ months". In addition, the patient has to experience, at least, three of the following six symptoms including restlessness or feeling keyed up or on edge, being easily fatigued, difficulty concentrating or mind going blank, irritability, muscle tension, and sleep disturbance. There are different types of patients who may experience many of these symptoms; nevertheless, due to the DSM-IV requirement of excessive 
worries with symptoms being present for $>6$ months will not recognize this diagnosis despite experiencing substantial impairment in functioning (Bienvenu et al., 1998).

Overall, one of popular psychosocial treatments for the treatment of GAD is cognitive-behavioral therapy (CBT). The components of this therapy may be different to include the following: education about the symptoms and causes of anxiety, cognitive restructuring, Worry exposure, applied relaxation, increasing awareness, learning to monitor of anxious symptoms presenting as physical symptoms, and the automatic thoughts of worry created from situational and behavioral cues. Patients are taught to manage these symptoms through training in arousal reduction techniques (Roemer \& Orsillo, 2002).

Worry exposure (WE) is a core of cognitive-behavioral treatment for generalized anxiety disorder (GAD) (Borkovec \& Newman, 1999; Borkovec et al., 2004). Past meta-analyses evaluating the effectiveness of CBT for GAD implemented general measures of anxiety to evaluate symptom severity and improvement. However, these studies do not sufficient support on whether CBT substantially reduces the cardinal symptom of GAD: pathological worry. Covin et al. (2008) performed a meta-analysis to evaluate relevant outcome studies, including the use of the Penn State Worry Questionnaire as the primary outcome variable. They reported a large overall effect size (ES) moderated by age and modality of treatment. Specifically, the largest gains were detected for younger adults and for individual treatment. Analyses also disclosed overall maintenance of gains at 6- and 12-month follow-up.

According to Hoyer et al. (2009), WE is a core element of cognitive-behavioral treatment for GAD. Hoyer et al. (2009) examined whether WE alone is as efficacious as the empirically supported standalone treatment for GAD, applied relaxation (AR). They reported that a stand-alone exposure in sensu technique, WE, could be efficacious in the treatment of GAD. Kessler et al. (2005) investigated prevalence, severity, and comorbidity of 12-month DSM-IV disorders in the National Comorbidity Survey Replication.

Linden et al. (2004) performed a controlled clinical trial to evaluate the efficacy of CBT treatment in outpatients with pure GAD who were treated by a therapist working in routine care. They concluded that CBT could an effective technique of treatment for GAD and differences between control and treatment group were comparable to or larger than those reported in studies on antidepressant drugs. Liss et al. (2005) performed a sensory processing sensitivity and its relationship with parental bonding, anxiety, and depression.

Mitte (2005) performed a meta-analysis of cognitive-behavioral treatments for generalized anxiety disorder by performing a comparison with pharmacotherapy. Warner et al. (2009) executed a CBT for anxiety and associated somatic complaints in pediatric medical settings and used an open pilot study as a case study. Wells and King (2006) discussed metacognitive therapy for generalized anxiety disorder. Wolitzky-Taylor and Telch (2010) measured efficacy of self-administered treatments for pathological academic worry.

Alilou et al. (2011) compared the intolerance of uncertainty, cognitive avoidance, negative problem orientation and positive beliefs about worry in generalized anxiety disorder patients with normal individuals. Ballenger et al. (2001) discussed the views of the International Consensus Group on Depression and Anxiety and reported that cognitive-behavioral therapy was the preferred form of psychotherapy for GAD, although when GAD is comorbid with depression, pharmacotherapy is increasingly indicated (Beck et al., 1996; Bennett-Levy et al., 2005).

Davidson (2004) implemented benzodiazepines in social anxiety disorder, GAD, and posttraumatic stress disorder and proposed some suggestion to reduce the effect of social anxiety disorder. Dugas 
and Robichaud (2007) presented a comprehensive review on cognitive-behavioral treatment for generalized anxiety disorder and discussed the challenges are faced from science perspective to practice. Fisher (2007) discussed the efficacy of psychological treatment for generalized anxiety disorder. Hamidpour (2008) examined the efficacy and effectiveness of mindfulness - based cognitive therapy (Tyrer, 1999) in treatment and presentation of relapse and recurrence of dysthymia.

The organization of this paper is organized as follows. We first present details of our different tests we implement on three patients in section 2. Section 3 presents details of our computational results and finally concluding remarks are given in the last to summarize the contribution of this paper.

\section{The proposed method}

The present study examines the efficacy of WE in treating patients with GAD. Three patients with GAD were selected using Structured Clinical Interview for DSM-IV (SCID) based on disorders axis I. Table 1 shows personal characteristics of the patients.

\section{Table 1}

Personal characteristics of the patients

\begin{tabular}{llllll}
\hline Group & Gender & Age & Marital status & Level of Educations & Medication \\
A & Female & 45 & Married & Master's degree & Drug medication \\
B & Female & 36 & Single & Diploma & No medication \\
C & Male & 24 & Single & Bachelor of science & Drug medication \\
\hline
\end{tabular}

Subjects are selected using purposeful sampling, and underwent the treatment after gaining treatment needs. Multiple baseline experimental single case study is then used as the method of the present study. The treatment program is carried out for 8 weekly sessions, with a follow up period of 3 months later treatment ending. Subjects completed Pennsylvania State Worry Questionnaire (PSWQ) and Cognitive Avoidance Questionnaire (CAQ).

\section{The results}

In this section, we present details of our findings on the experiments. Table 2 demonstrates the results of PSWQ and CAQ on three samples.

\section{Table 2}

The summary of the results of PSWQ and CAQ on three samples

\begin{tabular}{lccc}
\hline & A & B & C \\
\hline PSWQ (based-line) & 70 & 65 & 63 \\
PSWQ (first session) & 96 & 62 & 60 \\
PSWQ (fourth session) & 52 & 47 & 41 \\
PSWQ (eighth session) & 42 & 32 & 37 \\
\% of recovery & $40 \%$ & $51 \%$ & $\mathbf{4 1 \%}$ \\
Total \% of recovery & & $\mathbf{4 4 \%}$ & 77 \\
CAQ (based-line) & 94 & 80 & 74 \\
CAQ (first session) & 91 & 79 & 55 \\
CAQ (fourth session) & 63 & 49 & 40 \\
CAQ (eighth session) & 52 & 43 & $48 \%$ \\
\% of recovery & $52 \%$ & $46 \%$ & \\
Total \% of recovery & & $\mathbf{4 6 \%}$ & \\
\hline
\end{tabular}

In order to have a better understanding on comparing the effect of different tests on three patients, we have depicted them and Fig. 1 demonstrates the results of PSWQ and CAQ tests. 

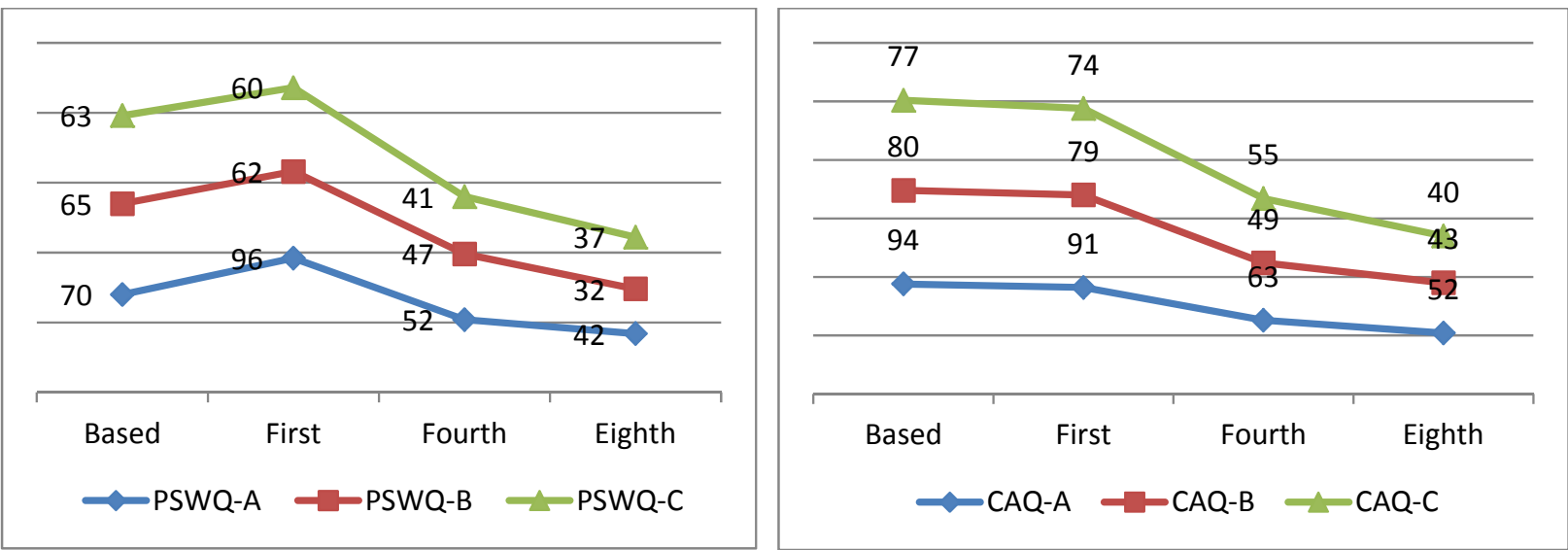

Fig. 1. The results of PSWQ and CAQ tests

As we can observe from the results of Table 2 and Fig. 1, the level of PSWQ and CAQ among three patients have been reduced after the eighth sessions. We have measured the level of STAI-T and BDI-II for all three patients and the results are summarized in Fig. 2 and Table 3.

Table 3

The summary of the results of STAI-T and BDI-II on three samples

\begin{tabular}{lccc}
\hline & A & B & C \\
\hline STAI-T (based-line) & 52 & 51 & 52 \\
STAI-T (first session) & 49 & 50 & 49 \\
STAI-T (fourth session) & 41 & 31 & 41 \\
STAI-T (eighth session) & 28 & 21 & 28 \\
\% of recovery & $46 \%$ & $59 \%$ & $46 \%$ \\
Total \% of recovery & & $\mathbf{5 2 \%}$ & 29 \\
BDI-II (based-line) & 29 & 34 & 26 \\
BDI-II (first session) & 26 & 33 & 16 \\
BDI-II (fourth session) & 16 & 21 & 10 \\
BDI-II (eighth session) & 10 & 15 & $66 \%$ \\
\% of recovery & $66 \%$ & $56 \%$ & \\
Total \% of recovery & & $61 \%$ & \\
\hline
\end{tabular}
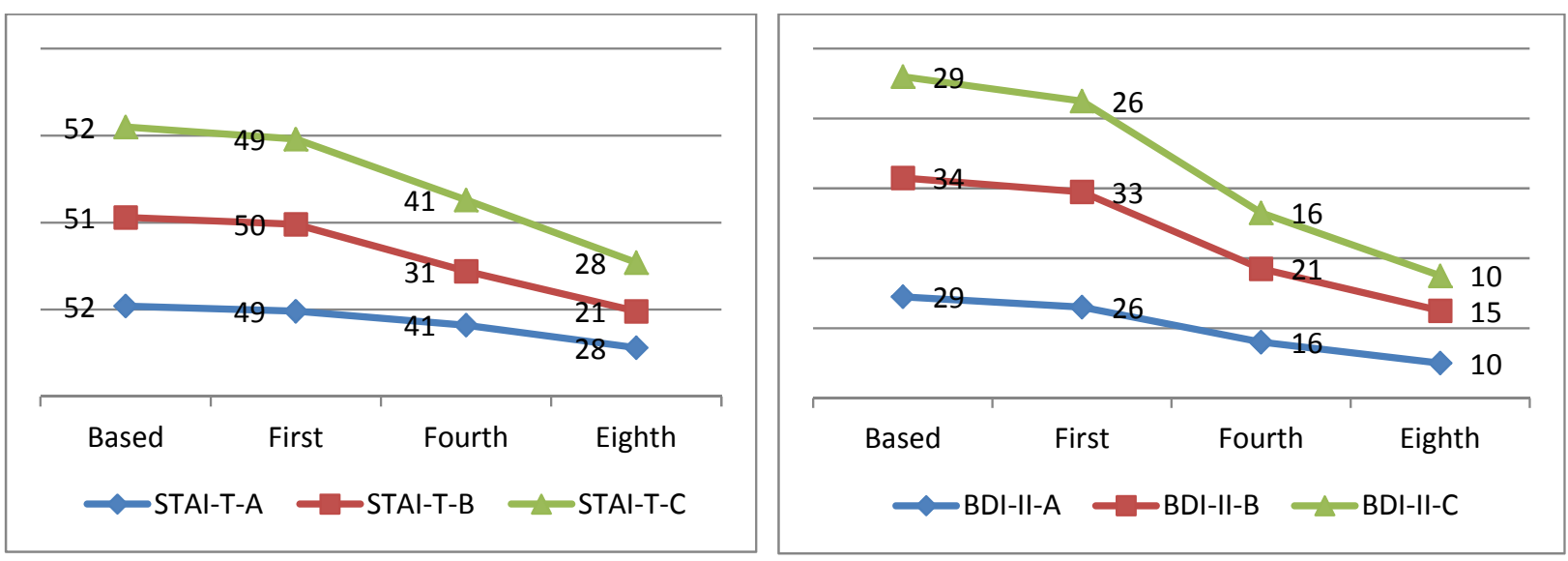

Fig. 2. The results of STAI-T and BDI-II tests 
Again, we can observe from the results of Table 3 and Fig. 3, the level of STAI-T and BDI-II among three patients have been reduced after the eighth sessions.

\section{Conclusion}

This is the second study investigating the efficacy of WE as stand-alone treatment for patients with DSM-IV GAD. The patients demonstrated distinct improvements on all outcome measures in active treatment conditions. Therefore, symptoms of anxiety and depression, excessive worrying and Cognitive Avoidance were reduced. These improvements appeared stabile, as marked by 3-month follow-up assessments. One particularly interesting finding was that all secondary outcome measures designed to evaluate cognitive change were efficiently reduced despite the fact that no clear cognitive intervention was applied. These results are consistent with other studies that question the necessity of clear cognitive interventions. Although the assumed pathways through which WE brought about change clearly differ, they may have a higher-level form in common. For instance, treatment enable the patient to feel more competent when confronted with upcoming worries.

This form of competency is often described as self-efficacy and may indirectly impact related (negative) beliefs about worrying as well the necessity to control one's own thinking. Our analyses did not disclose any moderating impact of psychiatric comorbidity on the treatment outcome, as the improvements were maintained even after controlling for another comorbid disorders. However, this does not mean that comorbid disorders are neutral for the therapeutic effect. Moderate and severe forms of depression are seen as a contraindication for WE and patients reaching the respective degree of severity of depression were excluded from the present study. Consequently, our results only confirm the view that WE can be applied successfully when mild depression or other comorbid conditions such as anxiety disorders are present. Furthermore, other potential moderating such as treatment expectations or the quality of the therapeutic alliance where not found to influence the results in a significant way.

In summary, in this second trial of WE as a stand-alone treatment for GAD we found this method to be efficacious. Although WE represent effective principles of change in GAD, this treatment should be further developed more systematically combined with other treatment components.

\section{Acknowledgment}

The authors would like to thank the anonymous referees for constructive comments on earlier version of this work.

\section{References}

Alilou, M., Shahjooyi T, \& Hashemi Z. (2011). The Comparison of intolerance of uncertainty, cognitive avoidance, negative problem orientation and positive beliefs about worry in generalized anxiety disorder patients with normal individuals. Journal of Tabriz University Psychology, 5(20), [In Persian].

Ballenger, J. C., Davidson, J. R., Lecrubier, Y., Nutt, D. J., Borkovec, T. D., Rickels, K., ... \& Wittchen, H. U. (2001). Consensus statement on generalized anxiety disorder from the International Consensus Group on Depression and Anxiety. Journal of Clinical Psychiatry. 62(11), 53-58. 
Beck, A. T., Steer, R. A., Ball, R., \& Ranieri, W. F. (1996). Comparison of Beck Depression Inventories-IA and-II in psychiatric outpatients. Journal of personality assessment, 67(3), 588597.

Bennett-Levy, J., Butler, G., Fennell, M., Hackmann, A., Mueller, M., \& Westbrook, D. (2005). Oxford guide to behavioural experiments in cognitive therapy. Oxford University Press.

Bienvenu, O. J., Nestadt, G., \& Eaton, W. W. (1998). Characterizing generalized anxiety: temporal and symptomatic thresholds. The Journal of Nervous and Mental Disease, 186(1), 51-56.

Borkovec, T. D., \& Newman, M. G. (1999). Worry and generalized anxiety disorder. Comprehensive clinical psychology, 6, 439-459.

Borkovec, T. D., Alcaine, O., \& Behar, E. (2004). Avoidance theory of worry and generalized anxiety disorder. Generalized anxiety disorder: Advances in research and practice.

Covin, R., Ouimet, A. J., Seeds, P. M., \& Dozois, D. J. (2008). A meta-analysis of CBT for pathological worry among clients with GAD. Journal of Anxiety Disorders, 22(1), 108-116.

Davidson, J.R.T. (2004). Use of benzodiazepines in social anxiety disorder, GAD, and posttraumatic stress disorder. Journal of clinical psychiatry, 65, 29-33.

Dugas, M. J., \& Robichaud, M. (2007). Cognitive-behavioral treatment for generalized anxiety disorder: From science to practice. Brunner-Routledge.

Fata L. (2004). The structures of meaning of the emotional states and Cognitive processing of emotional information: Comparison of two Conceptual Framework [Unpublished Ph.D. Dissertation]: Iran University of Medical Sciences,[In Persian].

Fisher P. (2007). The efficacy of psychological treatment for generalized anxiety disorder. In. G. C. L. Davey A. Wells. (Eds.), Worry and its psychological disorder. New York: Wiley. 356-77.

Hamidpour, H. (2008). Examination of efficacy and effectiveness of mindfulness - based cognitive therapy in treatment and presentation of relapse and recurrence of dysthymia. Journal of Research on Psychological Health, 1(1), 25-36 [In Persian].

Hoyer, J., Beesdo, K., Gloster, A. T., Runge, J., Höfler, M., \& Becker, E. S. (2009). Worry exposure versus applied relaxation in the treatment of generalized anxiety disorder. Psychotherapy and Psychosomatics, 78(2), 106-115.

Kessler, R. C., Chiu, W. T., Demler, O., \& Walters, E. E. (2005). Prevalence, severity, and comorbidity of 12-month DSM-IV disorders in the National Comorbidity Survey Replication. Archives of general psychiatry, 62(6), 617.

Linden, M., Zubraegel, D., Baer, T., Franke, U., \& Schlattmann, P. (2004). Efficacy of cognitive behaviour therapy in generalized anxiety disorders. Psychotherapy and psychosomatics, 74(1), 3642.

Liss, M., Timmel, L., Baxley, K., \& Killingsworth, P. (2005). Sensory processing sensitivity and its relation to parental bonding, anxiety, and depression. Personality and Individual Differences, 39(8), 1429-1439.

Mitte, K. (2005). Meta-analysis of cognitive-behavioral treatments for generalized anxiety disorder: a comparison with pharmacotherapy. Psychological Bulletin, 131(5), 785.

Roemer, L., \& Orsillo, S. M. (2002). Expanding our conceptualization of and treatment for generalized anxiety disorder: Integrating mindfulness/acceptance-based approaches with existing cognitive-behavioral models. Clinical Psychology: Science and Practice, 9(1), 54-68.

Tyrer, P. (1999). Anxiety: A multidisciplinary review. London: Imperial College Press.

Warner, C. M., Reigada, L. C., Fisher, P. H., Saborsky, A. L., \& Benkov, K. J. (2009). CBT for anxiety and associated somatic complaints in pediatric medical settings: an open pilot study. Journal of clinical psychology in medical settings,16(2), 169-177.

Wells, A., \& King, P. (2006). Metacognitive therapy for generalized anxiety disorder: An open trial. Journal of Behavior Therapy and Experimental Psychiatry, 37(3), 206-212.

Wolitzky-Taylor, K. B., \& Telch, M. J. (2010). Efficacy of self-administered treatments for pathological academic worry: A randomized controlled trial. Behaviour research and therapy, 48(9), 840-850. 